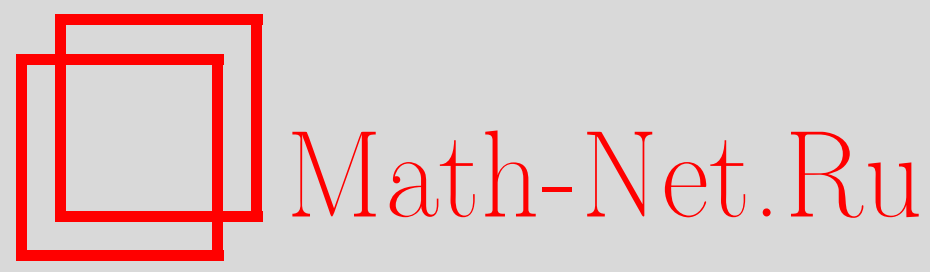

Г. А. Рудых, А. В. Синицын, О разрешимости нелинейной краевой задачи, возникающей при моделировании диффузии плазмы поперек магнитного поля и ее равновесных конфигураций, Матем. заметки, 2005, том 77, выпуск 2, 219234

DOI: https://doi.org/10.4213/mzm2486

Использование Общероссийского математического портала Math-Net.Ru подразумевает, что вы прочитали и согласны с пользовательским соглашением http://www.mathnet.ru/rus/agreement

Параметры загрузки:

IP : 54.237 .206 .68

26 апреля 2023 г., 12:39:22

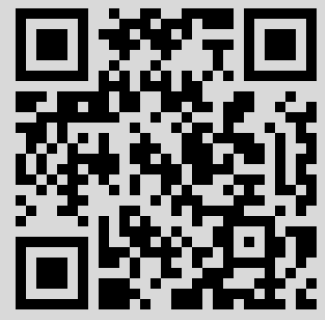




\title{
О РАЗРЕШИМОСТИ НЕЛИНЕЙНОЙ КРАЕВОЙ ЗАДАЧИ, ВОЗНИКАЮЩЕЙ ПРИ МОДЕЛИРОВАНИИ ДИФФУЗИИ ПЛАЗМЫ ПОПЕРЕК МАГНИТНОГО ПОЛЯ И ЕЕ РАВНОВЕСНЫХ КОНФИГУРАЦИЙ
}

\author{
Г. А. Рудых, А. В. Синицын
}

\begin{abstract}
Изучается простейшая одномерная модель баланса плотностей плазмы в установке типа токамак, сводящаяся к начально-краевой задаче для параболического уравнения второго порядка с неявным вырождением, содержащего нелокальные (интегральные) операторы. Задача о стабилизации нестационарных решений к стационарным сведена к исследованию разрешимости нелинейной интегро-дифференциальной краевой задачи. Получены достаточные условия на параметры изучаемой краевой задачи, обеспечивающие существование и единственность классического стационарного решения, для которого конструктивно построена область притяжения.
\end{abstract}

Библиограбоия: 26 названий.

1. Введение. Диффузия плазмы через магнитное поле изучалась в работах [1]-[5] и описывается в общем случае нелинейньми вырождающимися параболическими уравнениями второго порядка [6]. Фундаментальные результаты, полученные в работах [7], [8], являются основополагающими при математическом моделировании как медленной, так и быстрой диффузии плазмы через магнитное поле. В настоящее время имеется значительное число публикаций [6], [9]-[13], посвященных исследованию параболических уравнений с неявным вырождением вида

$$
\begin{gathered}
u_{t}=\Delta g(u)+f(\lambda, u), \quad(t, x) \in \mathbb{R}^{+} \times \Omega, \\
u=0, \quad(t, x) \in \mathbb{R}^{+} \times \partial \Omega, \quad u=u_{0}, \quad(t, x) \in\{0\} \times \Omega,
\end{gathered}
$$

где $\mathbb{R}^{+}=(0, \infty), \Omega \subset \mathbb{R}^{n}$ - открытое ограниченное подмножество с границей $\partial \Omega$ класса $C^{2+\alpha}, \alpha \in(0,1), g: \overline{\mathbb{R}}^{+} \rightarrow \overline{\mathbb{R}}^{+}{ }_{-}$непрерывная возрастающая функция, $g(0)=0, g^{-1}$ непрерьвна по Гёльдеру, $g$ или $g^{-1}$ локально непрерьвна по Липшицу, $f: \mathbb{R} \times \overline{\mathbb{R}}^{+} \rightarrow \mathbb{R}-$ непрерьвная функция, $f(\lambda, \cdot)$ локально непрерывна по Липшицу, $f(\lambda, 0)=0$ для $\lambda \in \mathbb{R}$, $u_{0} \in L_{+}^{\infty}(\Omega)=\left\{u \in L^{\infty}(\Omega): u(x) \geqslant 0\right.$ для почти всех $\left.x \in \Omega\right\}$.

Работа выполнена при частичной финансовой поддержке фонда INTAS, грант INTAS-OPEN № 2000-15. 
ОПРЕДЕЛЕНИЕ 1. Под решением задачи (1.1) на $[0, T], T>0$, понимается функция

$$
u \in C\left([0, T] ; L_{1}(\Omega)\right) \cap L^{\infty}\left(Q_{T}\right), \quad Q_{T}=\Omega \times(0, T]
$$

такая, что

$$
\int_{\Omega} u(t) \varphi(t) d x-\iint_{Q_{t}}\left(u \varphi_{t}+g(u) \Delta \varphi\right) d x d t=\int_{\Omega} u_{0} \varphi(0) d x+\iint_{Q_{t}} f(\lambda, u) \varphi d x d t
$$

для всех $\varphi \in C^{2}\left(\bar{Q}_{t}\right), \varphi \geqslant 0, \varphi=0$ на $\Sigma_{T}=\partial \Omega \times(0, T], t \in[0, T]$.

Под решением задачи $(1.1)$ на $[0, \infty)$ понимается решение последней на $[0, T]$ для любого $T>0$. Верхнее (соответственно нижнее) решение начально-краевой задачи (1.1) определяется аналогично, посредством замены в соотношении (1.2) знака равенства на знак неравенства $\geqslant($ соответственно $\leqslant)$.

Под решением стационарной задачи

$$
-\Delta g(u)=f(\lambda, u), \quad x \in \Omega, \quad u=0, \quad x \in \partial \Omega
$$

понимается функция $u \in L^{\infty}(\Omega)$ такая, что

$$
-\int_{\Omega} g(u) \Delta \varphi d x=\int_{\Omega} f(\lambda, u) \varphi d x, \quad \lambda \in \mathbb{R}
$$

для любой функции $\varphi \in C^{2}(\bar{\Omega}), \varphi \geqslant 0$ в $\Omega, \varphi=0$ на $\partial \Omega$. Аналогично, как и для (1.1), определяются верхнее и нижнее решения краевой задачи (1.3). Так как $g^{-1}$ непрерьвна по Гёльдеру, то любое решение задачи (1.3) является классическим в том смысле, что $v=g(u) \in C^{2+\alpha}(\bar{\Omega})$ - классическое решение краевой задачи

$$
-\Delta v=h(\lambda, v), \quad x \in \Omega, \quad v=0, \quad x \in \partial \Omega,
$$

где $h: \mathbb{R} \times \overline{\mathbb{R}}^{+} \rightarrow \mathbb{R}$

$$
h(\lambda, v)=f\left(\lambda, g^{-1}(v)\right)=f(\lambda, \cdot) \circ g^{-1}(v)
$$

Вопросы стабилизации неотрицательных решений задачи (1.1) к стационарньм изучались многими авторами (см., например, [12], [13], а также [6], [10] и имеющиеся там ссылки).

Теорема А [12]. Начально-краевая задача (1.1) имеет единственное для $t \in$ $[0, T]$ решение $u\left(u_{0}, t\right)$. Кроме того, $u\left(u_{0}, t\right) \geqslant 0$ для $t \in[0, T]$. 
Теорема В [12]. Пусть $u_{1} \in L_{+}^{\infty}(\Omega)$ (соответственно $u_{2} \in L_{+}^{\infty}(\Omega)$ ) - нижнее (соответственно верхнее) решение стачионарной задачи (1.3), $0 \leqslant u_{1} \leqslant u_{2}$. Тогда соответствующее решение $u\left(u_{1}, t\right)$ (соответственно $\left.u\left(u_{2}, t\right)\right)$ нестационарной задачи (1.1) является неубывающей (соответственно невозрастающей) функиией

$$
u_{1} \leqslant u\left(u_{1}, t\right) \leqslant u\left(u_{2}, t\right) \leqslant u_{2}, \quad t \in \overline{\mathbb{R}}^{+},
$$

$u$ u $\left(u_{1}, t\right), u\left(u_{2}, t\right)$ сходятся $п р и t \rightarrow+\infty\left(\right.$ в $C(\bar{\Omega})$, если $\operatorname{dim} \Omega=1$, или в $L^{p}(\Omega), p \geqslant 1$, если $\operatorname{dim} \Omega \geqslant 2$ ) монотонно соответственно $к$ пределам $u_{*}, u^{*}$, которые являются соответственно минимальным и максимальным стационарными решениями задачи (1.3) на множестве

$$
K=\left\{u \in L_{+}^{\infty}(\Omega): 0 \leqslant u_{1} \leqslant u \leqslant u_{2}\right\}
$$

Итак, асимптотическое поведение решений начально-краевой задачи (1.1) определяется множеством ее стационарных решений и связано с изучением его структуры.

Тем самым, задача об устойчивости стационарных решений сводится к исследованию структуры множества этих решений. В случае, когда удается доказать единственность стационарного решения, верхнее и нижнее решения задают область притяжения в пространстве начальных данных. Однако в настоящее время, как известно [14]-[16], не существует достаточно общих критериев единственности неотрицательных решений задач вида (1.4). Единственность можно гарантировать, если отображение $f(\lambda, \cdot) \circ g^{-1}(v)$ монотонно.

Наконец, отметим, что основными методами исследования уравнений вида (1.4) являются [17]-[19]: метод обькновенных дифференциальных уравнений, вариационные методы, метод верхних и нижних решений, метод априорных оценок и так называемьй метод теорем типа Лиувилля.

В этой работе исследуется разрешимость нелинейного операторного уравнения

$$
L u=F(x, u, z), \quad u \in D(L)
$$

с граничными условиями общего вида, входящими в оператор $L$, где $L$ - непрерывно обратимый дифференциальньй оператор, $F: \Omega \times \mathbb{R}^{1} \times \mathbb{R}^{1} \rightarrow \mathbb{R}$ - непрерывная функция своих аргументов, $\Omega \subset \mathbb{R}^{1}, z=T u, T$ - непрерьвньй нелинейный оператор (возможно, что $T$ - интегральный оператор типа Вольтерра или Фредгольма), действующий из $C(\bar{\Omega})$ в $C(\bar{\Omega}), F(x, \cdot, z)$ локально непрерывна по Липшицу и $F(x, u, T(\cdot))$ - монотонньй оператор.

В качестве $L$ можно рассматривать, например, либо равномерно эллиптический, либо обыкновенный дифференциальньй оператор второго порядка. Однако предложенный нами подход применим и к параболическим операторам. При этих предположениях доказьвается существование классического решения задачи (1.5). Помимо этого, получены достаточные условия, обеспечиваюшие единственность ее решения.

При получении этих результатов мы используем модификацию классических методов теории монотонных операторов в частично упорядоченных пространствах [14], [15] для случая парных неподвижных точек [16] в сочетании с техникой верхних и нижних решений [12], [17]. 
2. Теорема существования решения краевой задачи. Рассмотрим краевую задачу (1.5), где $L$ - непрерьвно обратимьй дифференциальный оператор. Как отмечалось вьше, в качестве $L$ можно взять равномерно эллиптический оператор, определенный на ограниченной выпуклой области $\Omega \subset \mathbb{R}^{n}$, граница которой $\partial \Omega$ принадлежит классу $C^{2+\alpha}$ для некоторого $\alpha \in(0,1)$, т.е.

$$
\begin{aligned}
L u= & -\sum_{i, j=1}^{n} a_{i j}(x) \frac{\partial^{2} u}{\partial x_{i} \partial x_{j}}+\sum_{i=1}^{n} b_{i}(x) \frac{\partial u}{\partial x_{i}}+c(x) u \\
& \sum_{i, j=1}^{n} a_{i j}(x) \xi_{i} \xi_{j} \geqslant \mu|\xi|^{2}, \quad \sum_{i=1}^{n} a_{i i}(x) \leqslant \nu
\end{aligned}
$$

для всех $x \in \Omega$, где $\xi=\left(\xi_{1}, \ldots, \xi_{n}\right) \in \mathbb{R}^{n}, \mu, \nu \in \mathbb{R}^{+}$- константы эллиптичности, $a_{i j}(x), b_{i}(x), c(x) \in C^{\alpha}(\bar{\Omega}), c(x) \geqslant 0$. Краевая задача $(1.5),(2.1)$ является базовой при математическом моделировании равновесных конфигураций ограниченной плазмы в установках типа токамак [20], [21].

В этом пункте изучается разрешимость двухточечной краевой задачи для обькновенного дифференциального оператора

$$
L u \equiv-\left(a(x) u^{\prime}\right)^{\prime}+b(x) u=F(x, u, z), \quad x \in(0,1), \quad a(x) \geqslant 0, \quad b(x) \geqslant 0,
$$

с граничньми условиями

$$
L_{i} u \equiv \alpha_{i} u(i)+(-1)^{i+1} \beta_{i} u^{\prime}(i)=\gamma_{i},
$$

где $\alpha_{i}, \beta_{i}, \gamma_{i} \in \mathbb{R}, i=0,1$. Пусть $F-$ непрерьвная функция, $z=T u$ - непрерьвный, возможно, нелинейный оператор, действующий из $C[0,1]$ в $C[0,1]$, где $C[0,1]-$ пространство вещественнозначных непрерывных функций c sup-нормой. Ниже предполагается, что $T$ - монотонный оператор, т.е. для любых $\varphi(x), \psi(x) \in C[0,1]$ таких, что $0 \leqslant \varphi(x) \leqslant \psi(x)$, имеем $T \varphi \leqslant T \psi$, если $T$ - возрастающий оператор, либо $T \varphi \geqslant T \psi$, если $T$ - убывающий оператор. Для определенности будем считать, что $T$ - убьвающий оператор. Наконец, отметим, что предлагаемьй ниже метод решения краевой задачи (1.5) применим также, если $z=z\left(T_{1} u, \ldots, T_{k} u\right), z: \mathbb{R}^{k} \rightarrow \mathbb{R}$, где операторы $T_{i}$ обладают приведенньми выше свойствами, причем тип монотонности (возрастание или убывание), может быть различным для различных $i=1,2, \ldots, k$.

Пусть заданы функции $v_{0}(x), w_{0}(x) \in C[0,1]$ такие, что $0 \leqslant v_{0}(x) \leqslant w_{0}(x)$. Посредством $\left[v_{0}, w_{0}\right]$ будем обозначать множество $\left\{u(x) \in C[0,1]: v_{0}(x) \leqslant u(x) \leqslant w_{0}(x)\right\}$ и назьвать его порядковым интервалом [22].

Предположим, что функция $F(x, u, z)$ для всех $u \in\left[v_{0}, w_{0}\right], z \in\left[T w_{0}, T v_{0}\right], x \in(0,1)$ обладает следующими свойствами:

(1) $F$ не убывает по $z$;

(2) $F(x, u, z)$ непрерывно дифференцируема по $u$ на порядковом интервале $\left[v_{0}, w_{0}\right]$;

(3) для любого $\tau \in(0,1)$ имеет место неравенство

$$
\tau F(x, u, T(\tau u))<F(x, \tau u, T u) .
$$


Отметим, что в силу условия (2) существует $M\left(v_{0}, w_{0}\right) \geqslant 0$ такое, что

$$
\frac{\partial}{\partial u} F \geqslant-M, \quad F+M u \geqslant 0 .
$$

Причем, если $F$ убьвает по $u$, то $M$ можно положить равной нулю.

ОПредЕЛЕниЕ 2. Решением задачи $(2.2),(2.3)$ назовем функцию $u(x) \in C^{2}(0,1] \cap$ $C^{1}[0,1]$, удовлетворяющую уравнению $(2.2)$ и граничным условиям $(2.3)$.

Пусть оператор $L$ непрерьвно обратим, тогда этим же свойством в силу неотрицательности $M$ обладает оператор $L+M$. Далее, предположим, что помимо (1)-(3) выполнено условие

(4) оператор $(L+M)^{-1}$ является $u_{0}$-положительным [14], [15], т.е. найдется такой ненулевой элемент $u_{0} \in C[0,1], u_{0}>0$, что для любого ненулевого элемента $u \in C[0,1], u>0$, можно указать числа $\alpha(u)>0, \beta(u)>0$, при которых вьполняется цепочка неравенств

$$
\alpha(u) u_{0} \leqslant(L+M)^{-1} u \leqslant \beta(u) u_{0} .
$$

ОПРЕДЕЛЕниЕ 3 . Функции $v_{0}, w_{0} \in C^{2}(0,1] \cap C^{1}[0,1]$, удовлетворяющие соотношениям

$L v_{0} \leqslant F\left(x, v_{0}, T w_{0}\right), \quad L_{i} v_{0}=0 \quad\left(\right.$ соответственно $\left.L w_{0} \geqslant F\left(x, w_{0}, T v_{0}\right), \quad L_{i} w_{0} \geqslant 0\right)$,

назовем нижним (соответственно верхним) квазирешениями задачи $(2.2),(2.3)$, где $i=0,1$.

Теорема 1. Пусть выполнено условие (1). Тогда краевая задача (2.2), (2.3) имеет, по крайней мере, одно решение и $(x)$, принадлежащее порядковому интервалу $\left[v_{0}, w_{0}\right]$.

ДокАЗАТЕЛЬСТво. Введем в рассмотрение вспомогательную функцию

$$
\begin{gathered}
H(x, u, T u) \equiv F(x, p(u), T p(u))+q(u), \quad x \in(0,1), \\
p(u)=\max \left\{v_{0}(x), \min \left\{u(x), w_{0}(x)\right\}\right\}, \quad q(u)= \begin{cases}\frac{w_{0}-u}{1+u^{2}}, & u \geqslant w_{0}, \\
0, & v_{0} \leqslant u \leqslant w_{0}, \\
\frac{v_{0}-u}{1+u^{2}}, & u \leqslant v_{0} .\end{cases}
\end{gathered}
$$

Из соотношений (2.5) следует справедливость включений

$$
p(u) \in\left[v_{0}, w_{0}\right], \quad T p(u) \in\left[T w_{0}, T v_{0}\right] .
$$

Далее, ясно, что функция $H(x, u, T u)$ непрерьвна и ограничена на множестве $(0,1) \times \mathbb{R}^{2}$. Тем самьм, по известному следствию теоремы Шаудера [22] краевая задача

$$
L u=H(x, u, T u), \quad L_{i} u=0
$$


имеет решение. Покажем, что при этом $u \in\left[v_{0}, w_{0}\right]$. С этой целью предположим, что найдутся $\varepsilon>0, x_{0} \in(0,1)$ такие, что $v_{0}\left(x_{0}\right)=u\left(x_{0}\right)+\varepsilon, v_{0}^{\prime}\left(x_{0}\right)=u^{\prime}\left(x_{0}\right)$, $v_{0}^{\prime \prime}\left(x_{0}\right) \leqslant u^{\prime \prime}\left(x_{0}\right)$. Тогда $p\left(u\left(x_{0}\right)\right)=v_{0}\left(x_{0}\right)$ и имеет место цепочка неравенств

$$
\begin{aligned}
\left.F\left(x, v_{0}, T w_{0}\right)\right|_{x=x_{0}} & \geqslant-a\left(x_{0}\right) v_{0}^{\prime \prime}\left(x_{0}\right) \geqslant-a\left(x_{0}\right) u^{\prime \prime}\left(x_{0}\right) \\
& =\left.F\left(x, v_{0}, T p(u)\right)\right|_{x=x_{0}}+\frac{\varepsilon}{1+u^{2}\left(x_{0}\right)} \\
& \geqslant\left. F\left(x, v_{0}, T w_{0}\right)\right|_{x=x_{0}}+\frac{\varepsilon}{1+u^{2}\left(x_{0}\right)} .
\end{aligned}
$$

Тем самым, в силу условия (1) и формул (2.6) приходим к противоречию. Следовательно, $u(x) \geqslant v_{0}(x), x \in(0,1)$. Аналогично доказьвается справедливость неравенства $u(x) \leqslant w_{0}(x)$. Теорема доказана.

3. Теорема единственности решения краевой задачи. Для доказательства единственности решения исследуемой краевой задачи $(2.2),(2.3)$ введем в рассмотрение линейное уравнение

$$
L u+M u=F(x, \eta, T \mu)+M \eta \equiv G(x, \eta, \mu),
$$

где $\eta, \mu \in C[0,1]$. Далее, определим отображение $A: C[0,1] \times C[0,1] \rightarrow C[0,1]$, которое ставит в соответствие каждой паре $(\eta, \mu) \in\left[v_{0}, w_{0}\right] \times\left[v_{0}, w_{0}\right]$ единственное решение

$$
u=A(\eta, \mu), \quad \eta, \mu \in C[0,1]
$$

краевой задачи $(3.1),(2.3)$.

УТВЕРЖДЕНИЕ 1. Пусть $A$ - отображсение, определяемое формулой (3.2). Пусть выполнены условия (2), (4). Тогда справедливы следующие свойства:

(a) $v_{0} \leqslant A\left(v_{0}, w_{0}\right), w_{0} \geqslant A\left(w_{0}, v_{0}\right)$;

(b) $A(\eta, \mu)$ не убывает по $\eta$ и не возрастает по $\mu$;

(c) $А$ является $u_{0}$-положительным.

ДокАЗАтЕльство. (а) Пусть $A\left(v_{0}, w_{0}\right)=v_{1}$. Покажем, что $v_{0} \leqslant v_{1}$. Действительно, если это неравенство не вьполняется, тогда существуют $\varepsilon>0, x_{0} \in(0,1)$ такие, что для функции $z=v_{0}-v_{1}$ имеем $z\left(x_{0}\right)=\varepsilon, z^{\prime}\left(x_{0}\right)=0, z^{\prime \prime}\left(x_{0}\right) \leqslant 0$. Тем самьд, мы приходим к противоречию: $0 \leqslant-a\left(x_{0}\right) z^{\prime \prime}\left(x_{0}\right) \leqslant M\left(v_{1}-v_{0}\right)\left(x_{0}\right)=-M \varepsilon$. Свойство (b) доказьвается аналогичными рассуждениями с использованием условия (2). Наконец, свойство (c) непосредственно следует из условия (4). Утверждение доказано.

ТеОрема 2. Пусть для $F:(0,1) \times \mathbb{R}^{2} \rightarrow \mathbb{R}$ выполнены условия (1)-(4). Тогда краевая задача $(2.2),(2.3)$ имеет единственное решение $и(x)$, принадлежсащее порядковому интервалу $\left[v_{0}, w_{0}\right]$. 
ДокАЗАТЕЛЬСтво. В первую очередь определим

$$
\begin{array}{ll}
(L+M) v_{n}=G\left(x, v_{n-1}, w_{n-1}\right), & L_{i} v_{n}=0, \\
(L+M) w_{n}=G\left(x, w_{n-1}, v_{n-1}\right), & L_{i} w_{n}=0,
\end{array}
$$

последовательные приближения $v_{n}, w_{n}$, где $n=1,2, \ldots$ Тогда имеет место цепочка неравенств

$$
v_{0} \leqslant v_{1} \leqslant \cdots \leqslant v_{n} \leqslant \cdots \leqslant w_{n} \leqslant \cdots \leqslant w_{1} \leqslant w_{0},
$$

причем $v_{n} \rightarrow u, w_{n} \rightarrow u$ равномерно при $n \rightarrow \infty$. Известно, что всякий порядковьй интервал $\left[v_{0}, w_{0}\right]$ является замкнутьм и вьпуклым множеством. Однако он не обязан быть ограниченным по норме, если порождающий упорядоченньй конус не является нормальным. Так как конус неотрицательных функций из $C[0,1]$ является нормальным $[14],[15]$, то $\left[v_{0}, w_{0}\right]$ будет ограниченным по норме. Тем самым, всякий порядковый интервал $\left[v_{0}, w_{0}\right]$ - ограниченное замкнутое и выпуклое множество. Итак, при наделении пространства $C[0,1]$ вещественнозначных непрерьвных функций sup-нормой и упорядочением, задаваемым конусом неотрицательных функций в $C[0,1]$, оператор $A=(L+M)^{-1}$, определяемьй формулой (3.2) и удовлетворяющий свойствам (a)-(c), согласно [16, теорема 2.1.7] является вполне непрерьвньм. Далее, введем в рассмотрение последовательность

$$
v_{n}=A\left(v_{n-1}, w_{n-1}\right), \quad w_{n}=A\left(w_{n-1}, v_{n-1}\right)
$$

Тем самым, из утверждения 1 следует, что $v_{0} \leqslant v_{1} \leqslant w_{1} \leqslant w_{0}$. Теперь предположим, что вьполняется цепочка неравенств

$$
v_{n-1} \leqslant v_{n} \leqslant w_{n} \leqslant w_{n-1} .
$$

Тогда из свойства (b) получим, что

$$
\begin{gathered}
v_{n}=A\left(v_{n-1}, w_{n-1}\right) \leqslant A\left(v_{n}, w_{n-1}\right) \leqslant A\left(v_{n}, w_{n}\right)=v_{n+1}, \\
w_{n}=A\left(w_{n-1}, v_{n-1}\right) \geqslant A\left(w_{n}, v_{n-1}\right) \geqslant A\left(w_{n}, v_{n}\right)=w_{n+1},
\end{gathered}
$$

откуда

$$
v_{n+1}=A\left(v_{n}, w_{n}\right) \leqslant A\left(w_{n}, w_{n}\right) \leqslant A\left(w_{n}, v_{n}\right)=w_{n+1} .
$$

Итак, по индукции следует справедливость (3.4).

Далее, в силу полной непрерывности оператора $A$ множество $\left\{v_{0}, v_{1}, v_{2}, \ldots\right\}$ является относительно компактным (предкомпактным). Тем самьм, найдется подпоследовательность $\left\{v_{n_{k}}\right\} \subset\left\{v_{n}\right\}$ такая, что $v_{n_{k}} \rightarrow v^{*}$. Ясно, что

$$
v_{n} \leqslant v^{*} \leqslant w_{n}, \quad n=1,2, \ldots, \quad 0 \leqslant v^{*}-v_{m} \leqslant v^{*}-v_{n_{k}} \text { для } m>n_{k} .
$$

Теперь, учитьвая, что конус неотрицательных функций в $C[0,1]$ является нормальным, имеем $\left\|v^{*}-v_{m}\right\| \leqslant\left\|v^{*}-v_{n_{k}}\right\| \rightarrow 0$. Итак, $v_{n} \rightarrow v^{*}, v^{*} \in\left[v_{0}, w_{0}\right]$. Совершенно аналогично можно доказать, что $w_{n} \rightarrow w^{*}, w^{*} \in\left[v_{0}, w_{0}\right]$. Далее, поскольку $A$ - деминепрерьвньй оператор, $A\left(v_{n}, w_{n}\right)$ слабо сходится к $A\left(v^{*}, w^{*}\right)$, т.е. $A\left(v_{n}, w_{n}\right) \rightarrow A\left(v^{*}, w^{*}\right)$. 
Также, очевидно, что $A\left(w_{n}, v_{n}\right) \rightarrow A\left(w^{*}, v^{*}\right)$. Таким образом, $v^{*}=A\left(v^{*}, w^{*}\right)$ и $w^{*}=$ $A\left(w^{*}, v^{*}\right)$, т.е. $\left(v^{*}, w^{*}\right)$ - парная неподвижная точка [16], [23].

Допустим, что $(\bar{v}, \bar{w})$ - другая парная неподвижная точка. Так как $v_{0} \leqslant \bar{v} \leqslant w_{0}$, $v_{0} \leqslant \bar{w} \leqslant w_{0}$

$$
\begin{gathered}
v_{1}=A\left(v_{0}, w_{0}\right) \leqslant A(\bar{v}, \bar{w})=\bar{v} \leqslant A\left(w_{0}, v_{0}\right)=w_{1}, \\
v_{1}=A\left(v_{0}, w_{0}\right) \leqslant A(\bar{w}, \bar{v})=\bar{w} \leqslant A\left(w_{0}, v_{0}\right)=w_{1},
\end{gathered}
$$

то аналогично по индукции можно доказать, что выполняются неравенства

$$
v_{n} \leqslant \bar{v} \leqslant w_{n}, \quad v_{n} \leqslant \bar{w} \leqslant w_{n}, \quad n=0,1,2, \ldots
$$

Из (3.5), взяв предел, получаем, что $v^{*} \leqslant \bar{v} \leqslant w^{*}, v^{*} \leqslant \bar{w} \leqslant w^{*}$. Тем самым, парная неподвижная точка $\left(v^{*}, w^{*}\right)$ обладает свойством максимальности и минимальности в том смысле, что для любой другой парной неподвижной точки $(\bar{v}, \bar{w})$ вьполняются выписанные вьше неравенства. Далее, из условия (3) и свойства (c) имеем, что для всех $\tau \in(0,1)$ справедлива цепочка неравенств

$$
A(\tau u, v)-\tau A(u, \tau v) \geqslant \alpha u_{0} \geqslant \frac{\alpha}{\beta} A(\tau u, v) .
$$

Теперь определим

$$
\tau_{0}=\inf \left\{\tau>0: v^{*} \geqslant \tau w^{*}\right\} .
$$

Очевидно, что $\tau_{0} \leqslant 1, v^{*} \geqslant \tau_{0} w^{*}$. Пусть $\tau_{0}<1$; тогда с учетом формулы $(3.6)$ и свойства (b) получим

$$
\begin{aligned}
\tau_{0} w^{*} & =\tau_{0} A\left(w^{*}, v^{*}\right) \leqslant \tau_{0} A\left(w^{*}, \tau_{0} w^{*}\right) \leqslant\left(1-\frac{\alpha}{\beta}\right) A\left(\tau_{0} w^{*}, w^{*}\right) \\
& \leqslant\left(1-\frac{\alpha}{\beta}\right) A\left(v^{*}, w^{*}\right)=\left(1-\frac{\alpha}{\beta}\right) v^{*}
\end{aligned}
$$

что противоречит определению $\tau_{0}$. Тем самьм, $\tau_{0}=1$. Поскольку по построению $v^{*} \leqslant w^{*}$, то $v^{*}=w^{*}=u, u=A(u, u)$. Наконец, из максимального и минимального свойств парной неподвижной точки $\left(v^{*}, w^{*}\right)$ следует единственность $u$. Теорема доказана.

4. Математическая модель баланса плотностей плазмы в установке типа токамак и свойства ее стационарных решений. В общем случае математическая модель, описьвающая эволюционньй процесс в тороидальной плазме, обладающей аксиальной симметрией, представляет собой двумерную задачу. Однако благодаря существенному различию (на несколько порядков) характерных времен переноса вдоль и поперек магнитных поверхностей ее удается достаточно хорошо описать в рамках одномерных моделей, получивших название транспортных [24]. Их основу составляет система диффузионньх уравнений [25], выражающих баланс частиц и энергии плазмы на каждой магнитной поверхности. Причем снижение размерности изучаемой задачи достигается за счет усреднения исследуемых величин и потоков по магнитным поверхностям, отражающего высокую скорость продольного перемещения. Наконец отметим, что 
диффузионные модели идеально приспособлены для изучения эволюции плазмы и полоидального магнитного поля в аксиально-симметричных тороидальных замкнутых конфигурациях типа токамак.

Рассмотрим простейшую транспортную модель баланса плотностей плазмы и нейтральных частиц в установке типа токамак

$$
\begin{gathered}
n_{t}=\frac{\alpha}{x}\left(x n n_{x}\right)_{x}+\gamma \rho n-n, \\
\left(x \rho_{k}\right)_{x}=(-1)^{k} x \rho_{k} n, \quad \rho=\rho_{0}+\rho_{1}, \quad k=0,1,
\end{gathered}
$$

с начальньгм условием $\left.n(x, t)\right|_{t=0}=n_{0}(x) \geqslant 0$ и граничньми условиями

$$
\begin{aligned}
\left.x n_{x}\right|_{x=0} & =\left.n\right|_{x=1}=0, \\
\left.x\left(\rho_{0}-\rho_{1}\right)\right|_{x=0} & =0,\left.\quad \rho_{0}\right|_{x=1}=1 .
\end{aligned}
$$

Здесь $n(x, t)$ - плотность плазмы, $\rho_{0}(x)$ и $\rho_{1}(x)$ - плотности летящих со стенок к оси и соответственно встречных инжектируемых нейтральных частиц, $\rho(x)$ - полная плотность нейтралов в плазме, $x$ - радиус магнитной поверхности, $\alpha>0$ - коэффициент диффузии плазмы поперек магнитного поля, $\gamma>0$ - мощность инжекции.

Исследуемая нелинейная начально-краевая задача (4.1)-(4.4) содержит одну пространственную переменную $x$ и соответствует цилиндру с трансляционной и круговой симметриями. Причем трансляционная симметрия отражает аксиальную симметрию исходного тора, а круговая - процедуру усреднения в перпендикулярном сечении. Кроме того, предполагается, что на поверхности, с которой происходит инжекция, задан поток нейтралов, а плотность плазмы равна нулю.

С одной стороны, (4.2) при $k=0$ запишется в виде

$$
\frac{\partial}{\partial x}\left(x \rho_{0}(x, t)\right)=x \rho_{0}(x, t) n(x, t)
$$

и, как нетрудно убедиться, приводит к зависимости

$$
\rho_{0}(x, t)=\frac{c_{0}(t)}{x} \cdot \exp \left(\int_{0}^{x} n(s, t) d s\right) .
$$

Причем в силу второго из условий (4.4) получим, что

$$
c_{0}(t)=\exp \left(-\int_{0}^{1} n(s, t) d s\right)
$$

т.е.

$$
\rho_{0}(x, t)=\frac{1}{x} \cdot \exp \left(-\int_{0}^{1} n(s, t) d s\right) \exp \left(\int_{0}^{x} n(s, t) d s\right) .
$$

С другой стороны, (4.2) при $k=1$ принимает вид

$$
\frac{\partial}{\partial x}\left(x \rho_{1}(x, t)\right)=-x \rho_{1}(x, t) n(x, t) .
$$


Из этого соотношения непосредственно следует справедливость выражения

$$
\rho_{1}(x, t)=\frac{c_{1}(t)}{x} \cdot \exp \left(-\int_{0}^{x} n(s, t) d s\right)
$$

При этом в силу первого из условий (4.4) и с учетом вида функции $\rho_{0}(x, t)$ получим, что

$$
c_{1}(t)=\exp \left(-\int_{0}^{1} n(s, t) d s\right)
$$

т.е.

$$
\rho_{1}(x, t)=\frac{1}{x} \cdot \exp \left(-\int_{0}^{1} n(s, t) d s\right) \exp \left(-\int_{0}^{x} n(s, t) d s\right) .
$$

Тем самьм, имеет место формула

$$
\rho(x, t)=\frac{2}{x} \cdot \exp \left(-\int_{0}^{1} n(s, t) d s\right) \operatorname{ch}\left(\int_{0}^{x} n(s, t) d s\right) .
$$

В итоге справедлив следующий результат: исключая из системы (4.1), (4.2) с учетом граничных условий (4.4) функцию $\rho$, приходим к уравнению

$$
n_{t}=\frac{\alpha}{x}\left(x n n_{x}\right)_{x}+\left[\frac{2 \gamma}{x} \exp \left(-\int_{0}^{1} n(s, t) d s\right) \operatorname{ch} \int_{0}^{x} n(s, t) d s-1\right] n
$$

Стационарный аналог уравнения (4.5)

$$
-\frac{\alpha}{x}\left(x n n^{\prime}\right)^{\prime}=\left[\frac{2 \gamma}{x} \exp \left(-\int_{0}^{1} n d s\right) \operatorname{ch} \int_{0}^{x} n d s-1\right] n
$$

нагруженного граничньми условиями (4.3), является предметом исследования настоящего пункта, где $n^{\prime}=(d / d x) n(x)$.

Итак, рассмотрим краевую задачу (4.6), (4.3)

$$
\begin{gathered}
-\left(x u^{\prime}\right)^{\prime}=\lambda(\xi T u-x) u^{1 / 2} \equiv \lambda F(x, u, T u), \\
\left.x u^{\prime}\right|_{x=0}=\left.u\right|_{x=1}=0
\end{gathered}
$$

где $u=n^{2}, \xi=2 \gamma, \lambda=2 / \alpha$,

$$
T u=\exp \left(-\int_{0}^{1} u^{1 / 2} d s\right) \operatorname{ch}\left(\int_{0}^{x} u^{1 / 2} d s\right)
$$

С использованием теоремы 2 покажем разрешимость исследуемой интегро-дифференциальной краевой задачи (4.7), (4.8).

Другими словами, построим нижнее и верхнее квазирешения $v_{0}, w_{0} \in C^{2}(0,1] \cap$ $C^{1}[0,1]$ такие, чтобы вьполнялись условия $(1)-(4)$.

Непосредственными вычислениями несложно проверить, что оператор $T u$, определяемьй согласно (4.8), является монотонно убьвающим, т.е. для любых $\varphi(x)$, 
$\psi(x) \in C[0,1]$, удовлетворяюших условию $0 \leqslant \varphi(x) \leqslant \psi(x)$, справедливо неравенство $T \varphi(x) \geqslant T \psi(x)$.

Действительно, так как $0 \leqslant \varphi(x) \leqslant \psi(x)$, приходим к справедливости неравенств

$$
\exp \left(\int_{0}^{x} \varphi^{1 / 2} d s\right) \leqslant \exp \left(\int_{0}^{x} \psi^{1 / 2} d s\right), \quad \exp \left(-\int_{0}^{x} \psi^{1 / 2} d s\right) \leqslant \exp \left(-\int_{0}^{x} \varphi^{1 / 2} d s\right) .
$$

Отсюда, очевидно, следует соотношение

$$
\exp \left(\int_{0}^{x} \varphi^{1 / 2} d s\right)+\exp \left(-\int_{0}^{x} \psi^{1 / 2} d s\right) \leqslant \exp \left(\int_{0}^{x} \psi^{1 / 2} d s\right)+\exp \left(-\int_{0}^{x} \varphi^{1 / 2} d s\right)
$$

Тем самьм, имеет место зависимость

$$
\operatorname{sh}\left(\int_{0}^{x} \varphi^{1 / 2} d s\right) \leqslant \operatorname{sh}\left(\int_{0}^{x} \psi^{1 / 2} d s\right)
$$

Далее, возводя в квадрат обе части этого неравенства и используя формулу $1+\operatorname{sh}^{2}(\cdot)=$ $\operatorname{ch}^{2}(\cdot)$, легко показать, что

$$
\operatorname{ch}\left(\int_{0}^{x} \varphi^{1 / 2} d s\right) \leqslant \operatorname{ch}\left(\int_{0}^{x} \psi^{1 / 2} d s\right)
$$

Также очевидно, что выполняются следующие соотношения:

$$
\begin{aligned}
& \exp \left(-\int_{0}^{x} \varphi^{1 / 2} d s\right) \geqslant \exp \left(-\int_{0}^{x} \psi^{1 / 2} d s\right) \\
& \exp \left(-\int_{0}^{1} \varphi^{1 / 2} d s\right) \geqslant \exp \left(-\int_{0}^{1} \psi^{1 / 2} d s\right)
\end{aligned}
$$

Теперь ясно, что в силу зависимости

$$
-\exp \left(-\int_{0}^{1} \varphi^{1 / 2} d s\right) \leqslant-\exp \left(-\int_{0}^{1} \psi^{1 / 2} d s\right)
$$

справедливо представление

$$
-\exp \left(-\int_{0}^{1} \varphi^{1 / 2} d s\right) \operatorname{ch}\left(\int_{0}^{x} \varphi^{1 / 2} d s\right) \leqslant-\exp \left(-\int_{0}^{1} \psi^{1 / 2} d s\right) \operatorname{ch}\left(\int_{0}^{x} \psi^{1 / 2} d s\right) .
$$

В итоге заключаем, что имеет место неравенство

$$
\exp \left(-\int_{0}^{1} \varphi^{1 / 2} d s\right) \operatorname{ch}\left(\int_{0}^{x} \varphi^{1 / 2} d s\right) \geqslant \exp \left(-\int_{0}^{1} \psi^{1 / 2} d s\right) \operatorname{ch}\left(\int_{0}^{x} \psi^{1 / 2} d s\right) .
$$

Отсюда в силу формулы (4.8) следует, что $T \varphi(x) \geqslant T \psi(x)$. Тем самьп, исследуемый оператор (4.8) является монотонно убывающим. 
Помимо этого $0 \leqslant T u(x) \leqslant 1$ для всех $u(x) \in C[0,1]$. Итак условие $(1)$ выполнено. Далее, так как $F(x, u, z) / u \rightarrow 0$ при $u \rightarrow \infty$ равномерно по $x$ и $z$, существует постоянная $a^{2}>0$ такая, что

$$
\frac{F\left(x, a^{2}, z\right)}{a^{2}} \leqslant \frac{\xi}{a} \leqslant \frac{1}{\lambda}
$$

где $z=T u$. Полагая $w_{0}=a^{2}$, имеем

$$
A\left(w_{0}, v_{0}\right)=\lambda \int_{0}^{1} G(x, s) F\left(s, w_{0}, T v_{0}\right) d s \leqslant a^{2} \int_{0}^{1} G(x, s) d s \leqslant a^{2}=w_{0}
$$

где $G(x, s)$ - функция Грина соответствующего дифференциального оператора, определяемая формулой

$$
G(x, s)= \begin{cases}-\ln x, & s \leqslant x \\ -\ln s, & x \leqslant s .\end{cases}
$$

Очевидно, что $w_{0}=a^{2}$ будет искомым верхним квазирешением, если $a \geqslant \xi \lambda$.

Теперь рассмотрим вспомогательную краевую задачу на собственные значения

$$
L z \equiv-\left(x z^{\prime}\right)^{\prime}=\eta x z, \quad 0<x<1,\left.\quad x z^{\prime}\right|_{x=0}=\left.z\right|_{x=1}=0 .
$$

Хорошо известно [26], что решение $z(x)$ задачи Штурма-Лиувилля (4.12) имеет вид $z(x)=J_{0}\left(\eta_{0}^{1 / 2} x\right)$, где $J_{0}\left(\eta_{0}^{1 / 2} x\right)$ - функция Бесселя, $\max J_{0}=1, J_{0}>0$ при $x \in[0,1)$, $\eta_{0}$ - главное собственное значение. Далее, предположим, что $v_{0}=\nu^{2} z$, где $\nu$-некоторая постоянная. Тогда, учитывая (4.12), приходим к справедливости цепочки неравенств

$$
-\left(x v_{0}^{\prime}\right)^{\prime}-\lambda\left(\xi T w_{0}-x\right) v_{0}^{1 / 2} \leqslant \eta_{0} \nu\left(\nu-\frac{\lambda}{\eta_{0}}\left(\xi e^{-a}-1\right)\right) \leqslant 0
$$

при условии, что

$$
\nu \leqslant \frac{\lambda\left(\xi e^{-a}-1\right)}{\eta_{0}}, \quad a \leqslant \ln \xi
$$

Итак, если постоянная $\nu$ является достаточно малой, то $v_{0}=\nu^{2} J_{0}\left(\eta_{0}^{1 / 2} x\right)$ - нижнее квазирешение. Так как $F(x, u, T u)=(\xi T u-x) u^{1 / 2}$, то условие $(3)$ будет выполнено, если мы покажем, что имеет место соотношение

$$
\tau F(x, u, T(\tau u))-F(x, \tau u, T u)=(\tau u)^{1 / 2} f(x, y, z, t)<0
$$

где

$$
\begin{aligned}
& f(x, y, z, t)=t\left(\xi e^{-t y} \operatorname{ch}(t z)-x\right)-\left(\xi e^{-y} \operatorname{ch}(z)-x\right), \\
& y=\int_{0}^{1} u^{1 / 2} d s, \quad z=\int_{0}^{x} u^{1 / 2} d s, \quad t=\tau^{1 / 2}, \quad y \leqslant a .
\end{aligned}
$$

С этой целью докажем вспомогательное 
УТВЕРЖДЕНИЕ 2. Пусть $\xi_{1}=\xi, \xi_{2}=\xi /(2-\xi) u x_{i}-$ вещественный корень уравнения

$$
\exp x_{i}=\xi_{i}\left(1-x_{i}\right), \quad i=1,2 .
$$

Пусть выполнено одно из условий:

(5) если $1<\xi \leqslant 2 /\left(1-e^{-2}\right)$, то $a \leqslant \min \left\{x_{1}, x_{2} / 2\right\}$ (при $\xi>2 x_{2}$ - наименьший из корней уравнения (4.16) для $i=2$ );

(6) если $\xi>2 /\left(1-e^{-2}\right)$, то $a \leqslant x_{1}$.

Тогда $f(x, y, z, t)<0$ для всех $t \in(0,1), x \in[0,1), 0 \leqslant z \leqslant y \leqslant a$.

ДокАЗАТЕЛЬСтво. Проведем исследование на экстремум функции (4.15). Несложно проверить, что $f_{x}>0, f_{y}=f_{z}=0$ лишь при $t=1, f(x, y, z, t) \rightarrow 0^{-}$при $t \rightarrow 1^{-}$. Причем $0 \leqslant z \leqslant y \leqslant a$. В связи с этим

$$
\max f(x, y, z, t)=\max \left\{f_{1}(t)=f(1, a, 0, t), f_{2}(t)=f(1, a, a, t), f_{3}(t)=f(1,0,0, t)\right\}
$$

Сразу же отметим, что $f_{3}(t)<0$ при $\xi>1$. Далее, уравнение $f_{1}^{\prime}(t)=0$ запишется в виде $e^{a t}=\xi(1-a t)$; оно совпадает с (4.16) для $i=1$ и имеет единственное решение $x_{1}=a t_{1}$ при всех $\xi>1$. Помимо этого, $f_{1}^{\prime \prime}\left(t_{1}\right)<0$. Аналогично, уравнение $f_{2}^{\prime}(t)=0$ запишется в виде $(2-\xi) e^{2 a t}=\xi(1-2 a t)$; оно совпадает с $(4.16)$ для $i=2$ и при $1<\xi \leqslant 2$ обладает единственным решением $x_{2}=2 a t_{2}$, при $2<\xi \leqslant 2 /\left(1-e^{-2}\right)$ - двумя решениями $1<x_{2}=2 a t_{2}<2<x_{3}$ и, наконец, при $\xi>2 /\left(1-e^{-2}\right)$ не имеет решений. Кроме того, $f_{2}^{\prime \prime}\left(t_{2}\right)<0$. Тем самьм, из условий $(5),(6)$ следует, что $t_{1} \geqslant 1, t_{2} \geqslant 1$. Итак, для всех $t \in(0,1), x \in[0,1]$ и $0 \leqslant z \leqslant y \leqslant a$ имеем $f(x, y, z, t)<0$. Утверждение доказано.

ЗАмЕчАниЕ 1. Более того, можно показать, что условия (5), (6), входящиев утверждение 2, являются также и необходимыми для выполнения неравенства

$$
f(x, y, z, t)<0 \quad \text { при всех } t \in(0,1), \quad x \in[0,1], \quad 0 \leqslant z \leqslant y \leqslant a .
$$

Тем самым, с учетом утверждения 2 получаем справедливость неравенства (4.14). Отсюда заключаем, что условие (3) выполнено.

Далее, поскольку $x_{1}=a t_{1}, a \leqslant \ln \xi$, то из (4.16) для $i=1$ получаем, что $x_{1} \leqslant \ln \xi$, где $\xi>1$. В итоге, используя утверждение 2 , приходим к справедливости цепочки неравенств

$$
0<\xi \exp (-a)-1 \leqslant \xi T u-x .
$$

Так как $F(x, u, T u)=(\xi T u-x) u^{1 / 2}$, в силу (4.17) непосредственно получаем, что свойство (2) имеет место при $M=0$.

Наконец, покажем, что условие (4) также выполняется. В связи с этим докажем

УТВЕРЖДЕНИЕ 3. Пусть $y(x) \geqslant 0$ для $x \in[0,1)$ u $y(x) \not \equiv 0$. Тогда существуют $\alpha, \beta \in \mathbb{R}^{+}$такие, что выполняется неравенство

$$
\alpha(1-x) \leqslant \int_{0}^{1} G(x, s) y(s) d s \leqslant \beta(1-x),
$$

əде $G(x, s)$ - функиия Грина, определяемая формулой (4.11). 
ДокАЗАТЕЛЬСтво. Справедливость этого утверждения будет следовать из леммы 7.1 в [14], если мы покажем, что найдется $\varepsilon=\varepsilon\left(\left[x_{1}, x_{2}\right]\right)>0$ такое, что для каждого множества $\left[x_{1}, x_{2}\right] \subset[0,1]$ положительной меры имеет место неравенство

$$
\int_{x_{1}}^{x_{2}} G(x, s) d s \geqslant \varepsilon \int_{0}^{1} G(x, s) d s .
$$

С одной стороны, пусть $0 \leqslant x \leqslant\left(x_{1}+x_{2}\right) / 2$; тогда

$$
\begin{aligned}
\int_{x_{1}}^{x_{2}} G(x, s) d s & \geqslant \int_{\left(x_{1}+x_{2}\right) / 2}^{x_{2}} G(x, s) d s=-\int_{\left(x_{1}+x_{2}\right) / 2}^{x_{2}} \ln s d s \\
& =-\left.(s \ln s-s)\right|_{\left(x_{1}+x_{2}\right) / 2} ^{x_{2}}=a .
\end{aligned}
$$

С другой стороны, пусть $\left(x_{1}+x_{2}\right) / 2 \leqslant x \leqslant 1$; тогда

$$
\int_{x_{1}}^{x_{2}} G(x, s) d s \geqslant \int_{x_{1}}^{\left(x_{1}+x_{2}\right) / 2} G(x, s) d s=-\ln x \int_{x_{1}}^{\left(x_{1}+x_{2}\right) / 2} d s=-b \ln x \geqslant b(1-x) .
$$

Итак, для всех $x \in[0,1], a>b$ выполняется следующая оценка:

$$
\int_{x_{1}}^{x_{2}} G(x, s) d s \geqslant \min \{a, b(1-x)\}=b(1-x)=b \int_{0}^{1} G(x, s) d s,
$$

а следовательно, и неравенство (4.19). Тем самым, приходим к справедливости соотношения (4.18). Утверждение доказано.

Ясно, что утверждение 3 означает вьполнение условия (4).

В итоге, суммируя полученные результаты, заключаем, что имеет место

Теорема 3. Пусть $v_{0}=\nu^{2} J_{0}\left(\eta_{0}^{1 / 2} x\right), w_{0}=a^{2}$ - нижнее и соответственно верхнее квазирешения, $0 \leqslant v_{0} \leqslant w_{0}$. Пусть $a \geqslant \xi \lambda$, виполняются неравенства (4.13) и постоянная а удовлетворяет условиям утверэдения 2. Тогда краевая задача (4.7), (4.8) обладает единственным решением $u(x) \in\left[v_{0}, w_{0}\right]$, монотонно убивающим по $x$. Пусть $n\left(n_{0}(x), t\right)$ - решение задачи (4.5), (4.3) с начальньм условием

$$
n\left(n_{0}(x), 0\right)=n_{0}(x), \quad n_{0}(x) \in\left[\nu J_{0}^{1 / 2}\left(\eta_{0}^{1 / 2} x\right), a\right], \quad n_{0}(x) \in L_{+}^{\infty}(0,1) .
$$

Toгдa

$$
\left|x^{1 / 2}\left(n\left(n_{0}(x), t\right)-u^{1 / 2}(x)\right)\right| \rightarrow 0 \quad \text { равномерно по } x \quad \text { при } t \rightarrow+\infty .
$$

ДоКАЗАТЕЛЬСТво следует из теорем В и 2. При этом в теореме В мы считаем, что $x\left(u^{2}\right)_{x} \in L_{+}^{\infty}(0,1)$. Монотонность стационарного решения $n(x), \nu J_{0}^{1 / 2}\left(\eta_{0}^{1 / 2} x\right) \leqslant$ $n(x) \leqslant a$, следует из оценки (4.17), зависимости $u=n^{2}$ и принципа максимума.

Итак, получены достаточные условия на параметры $\xi, \lambda$, при выполнении которых существует единственное стационарное решение $n(x)$ краевой задачи $(4.6),(4.3)$. Кроме того, в пространстве начальньх данных построена область притяжения стационарного решения.

Суммируя результаты этого пункта, сформулируем полезньй, как нам представляется, результат. 
УТВЕРЖДЕНИЕ 4. Пусть выполнены неравенства (4.13) и одно из условий (5) или (6), где $x_{1}, x_{2}$ - корни уравнения (4.16), $\xi_{1}=\xi, \xi_{2}=\xi /(2-\xi)$ (в случае $\xi>2 x_{2}-$ наименьший из корней уравнения (4.16)). Тогда краевая задача (4.6), (4.3) имеет единственное стационарное решение $n(x), \nu J_{0}^{1 / 2}\left(\eta_{0}^{1 / 2} x\right) \leqslant n(x) \leqslant a$, причем $n(x)$ монотонно убивает по $x$.

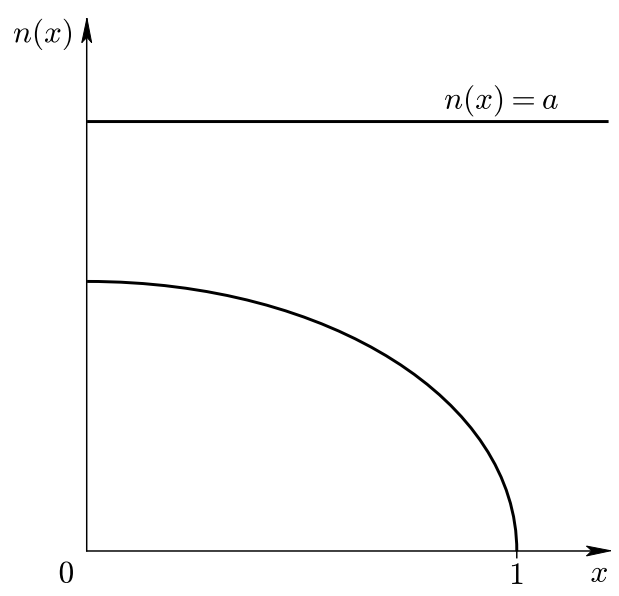

Рис. 1

Наконец, проведенные расчеты подтверждают заключения теоремы 3 (см. рис. 1).

Авторы выражают искреннюю благодарность рецензенту за полезные замечания, способствовавшие существенному улучшению нашей работы.

\section{СПИСОК ЦИТИРОВАННОЙ ЛИТЕРАТУРЫ}

[1] Hyman J., Rosenau P. Analysis of nonlinear parabolic equations modelling plasma diffusion across a magnetic field // Lectures in Appl. Math. 1986. V. 23. P. 219-245.

[2] Rosenau P., Hyman J. Plasma diffusion across a magnetic field // Phys. D. 1986. V. 20. P. 444-446.

[3] Rosenau P., Turkel E. Long time asymptotic of a system for plasma diffusion // TTSP. 1987. V. 16. № 2-3. P. 377-391.

[4] Kwong Y. Interior and boundary regularity of solutions to a plasma type equation // Proc. Amer. Math. Soc. 1988. V. 104. № 2. P. 472-478.

[5] Bertsch M., Kamin S. A system of degenerate parabolic equations // SIAM J. Math. Anal. 1990. V. 21. № 4. P. 905-916.

[6] Калашников А. С. Некоторые вопросы качественной теории нелинейных вырождающихся параболических уравнений второго порядка // УМН. 1987. Т. 42. № 2. С. 135-176.

[7] Олейник О.А., Калашников А. С., Чжоу-Юй-Линь. Задача Коши и краевые задачи для уравнений типа нестационарной фильтрации // Изв. АН СССР. Сер. матем. 1958. T. 22 . № 5. C. $667-704$.

[8] Сабинина Е. С. Об одном классе нелинейных вырождающихся параболических уравнений // Докл. АН СССР. 1962. Т. 143. № 4. С. 794-797.

[9] Галактионов В. А., Дородницын В. А., Еленин Г. Г., Курдюмов С. П., Самарский А. А. Квазилинейное уравнение теплопроводности: обострение, локализация, симметрия, точные решения, асимптотики, структуры // Современные проблемы матем. Новейшие достижения. Итоги науки и техники. Т. 28. М.: ВИНИТИ, 1987. С. 95-205. 
[10] Самарский А. А., Галактионов В. А., Курдюмов С. П., Михайлов А. П. Режимы с обострением в задачах для квазилинейных параболических уравнений. М.: Наука, 1987.

[11] Aronson D. G. Regularity of flows in porous media: a survey // Nonlinear Diffusion Equations and Their Equilibrium States. V. 1. New York: Springer-Verlag, 1988. P. 35-49.

[12] De Mottoni, Schiaffino A., Tesei A. Attractivity properties of nonnegative solutions for a class of nonlinear degenerate parabolic problems // Ann. Math. Pura Appl. 1984. V. 136. P. $35-48$.

[13] Aronson D. G., Crandall M. G., Peletier L. A. Stabilization of solutions of a degenerate nonlinear diffusion problem // Nonlinear Anal. 1982. V. 6. № 10. P. 1001-1022.

[14] Красносельский М. А. Положительные решения операторных уравнений. М.: Физматгиз, 1962.

[15] Красносельский М.А., Вайнико Г. М., Забрейко П. П., Рутицкий Я. Б., Стеценко В. Я. Приближенное решение операторных уравнений. М.: Наука, 1969.

[16] Guo D., Laksmikantham V. Nonlinear Problems in Abstract Cones. London: Academic Press, 1988.

[17] Похожаев С. И. Об уравнениях вида $\Delta u=f(x, u, D u) / /$ Матем. сб. 1980. Т. 113. № 2. C. $324-338$.

[18] Похожаев С. И. Об эллиптических задачах в $\mathbb{R}^{n}$ с суперкритическим показателем нелинейности // Матем. сб. 1991. Т. 182. № 4. С. 467-489.

[19] Митидиери Э., Похожаев С. И. Отсутствие глобальных положительных решений квазилинейных эллиптических неравенств // Докл. РАН. 1998. Т. 359. № 4. С. 456-460.

[20] Bandle C. A priori estimates and the boundary value of solutions for a problem arising in plasma physics // Nonlinear Anal. 1983. V. 7. № 4. P. 439-451.

[21] Rakotoson J. Un modéle non local en physique des plasmas: résolution par une méthode de degré topologique // Acta Appl. Math. 1985. V. 4. №1. P. 1-14.

[22] Хатсон В., Пим Дж. Приложения функционального анализа и теории операторов. М.: Мир, 1983.

[23] Guo D., Laksmikantham V. Coupled fixed points of nonlinear operator with applications // Nonlinear Anal. 1987. V. 11. № 5. P. 623-632.

[24] Днестровский Ю.Н., Костамаров Д. П. Математическое моделирование плазмы. М.: Наука, 1982.

[25] Хоган Дж. Т. Многокомпонентные модели переноса в токамаке // Вычислительные методы в физике. Управляемый термоядерный синтез. М.: Мир, 1980. С. 142-177.

[26] Владимиров В. С. Уравнения математической физики. М.: Наука, 1981.

Институт динамики систем и теории управления СО РАН, г. Иркутск

Поступило

E-mail: rudykh@icc.ru, avsin@icc.ru

21.08 .2002

Исправленный вариант

10.11.2003 